\title{
Molecular Docking and ADMET studies of Allium cepa, Azadirachta indica and Xylopia aethiopica isolates as potential anti-viral drugs for Covid-19
}

Peter Ifeoluwa Adegbola ( $\sim$ piadegbola27@lautech.edu.ng)

Ladoke Akintola University of Technology

\section{Banjo Semire}

Ladoke Akintola University of Technology

Olumide Samuel Fadahunsi

Ladoke Akintola University of Technology

Aanuoluwa Eunice Adegoke

Ladoke Akintola University of Technology

\section{Research Article}

Keywords: SAR-CoV-2, Plants, Docking, ADMET, Inhibitor

Posted Date: October 30th, 2020

DOI: https://doi.org/10.21203/rs.3.rs-100161/v1

License: (9) This work is licensed under a Creative Commons Attribution 4.0 International License.

Read Full License

Version of Record: A version of this preprint was published at VirusDisease on March 1st, 2021. See the published version at https://doi.org/10.1007/s13337-021-00682-7. 


\section{Abstract}

Purpose: Plants are repository of important constituents with proven efficacy against many human diseases including viral diseases. The antiviral activity of many plants including Azadirachta indica, Xylopia aethiopica and Allium cepa has been reported. The novel coronavirus disease is no exception among viral diseases in which plant compounds could serve as potent antagonist. Therefore, our study investigated the inhibitory potentials of Azadirachta indica and Xylopia aethiopica isolates against SARSCoV-2 viral accessory proteins and the host serine protease.

Methods: The protein data (SARS-CoV-2 Papain like protease (PLpro) (PDB: 6wx4), Chymotrypsin-like main protease (3CLpro) (PDB:6YB7), SARS-CoV nsp 12 (PDB: 6nus), Host cell protease (TMPRSS1) (PDB:5ce1) were obtained from the Protein Data Bank (PDB), while the SDS format of each Ligands were obtained from Pubchem database. Molecular docking analysis was performed with Auto Dock Vina 1.5.6 and visualization of the interaction between the ligands and protein was done with discovery studio 2019. The ADMET prediction of pharmacokinetics and toxicity properties of the ligands was obtained using vNN Web Server.

Results: Our result showed that all the plant isolates demonstrated negative Gibb's free energy, indicating good binding affinity for both the viral and host protein. Overall, twenty-three of the forty-seven isolates showed good binding affinity comparable with dexamethasone that was used as reference drug. Although many of the compounds have good binding affinity for the viral and host proteins, based on the ADMET prediction, only Azadironic acid, Nimbionone, Nimbionol and Nimocinol all from A. indica could serve as potential drug candidate with good pharmacokinetics and toxicity profile.

Conclusion: This study provides an insight into potential inhibitors and novel drug candidates for SARSCoV-2.

\section{Background}

Plant remains a dependable resort for drug discovery. They are repository of important compounds with varied biological functions. Many pharmacological active drugs have their origin from medicinal plants (Efferth and Koch, 2011; Zong et al., 2012). Among important plants with antiviral property are Azadirachta indica and Allium cepa. It has been reported that neem ( $A$. indica) seed extracts exhibited inhibitory property against Duck viral enteritis (Xu et al., 2012). In both in-vitro and in-vivo study, it showed inhibitory properties against Dengue virus type-2 (Parida et al., 2002). Two polysaccharides isolated from the leaves inhibited Poliovirus type 1 (Faccin-Galhardi et al., 2012). Organosulfur constituents of $A$. cepa are important candidate in the antiviral properties (Chen et al., 2011; Kumar and Pandy, 2013). Onion extracts block attachment of new castle disease virus to host cell (Harazem et al., 2019) and decrease the propagation of avian influenza virus in the embryonated chicken eggs (Ahmadi et al., 2018). In other words, these plants are promising source of antiviral drugs. 
The novel coronavirus diseases, which up until now is a pandemic with no sight of relive from its incidence. The virus up until date has claimed 646,641 lives and more than $15,114,449$ individuals have been infected globally (WHO, 2020). The coronavirus disease is no exception among viral diseases in which plant compounds can serve as potent antagonist. Going by the genomic structure of the virus and the behavior in the human host, drugs could be developed to target the host cells protein involved in viralhost interaction or directly on the viral accessory proteins responsible for viral pathogenesis (Wu et al., 2020). A number of research efforts are focused towards the discovery of potential drug candidates targeting one or more of the viral proteins involved in its virulence.

In this study, compounds previously identified in Allium cepa, Azadirachta indica and Xylopia aethiopica (Table 6) were docked against four target proteins of SARS-COV-2. The approach used in this study involved the identification of compounds that could directly target any of the proteins involved at different stages of viral pathogenesis or compounds with dual or multiple target potentials. In this regard, three of the proteins involved in viral RNA synthesis and replication (Papain-like protease (PLpro), 3C-like main protease (3CPLpro and RNA-dependent-RNA polymerase (RdRp), and host protein involved in viral entry were screened to identify potential inhibitors based on the formation of stable complex with ligands which exhibited more negative free energy of binding $(\Delta G)$ and low inhibition constant $(\mathrm{Ki})$. The interaction of the natural compounds with the viral and host proteins was visualized in other to predict compounds that may inhibit the novel coronavirus and provide information on potential compounds for therapeutic development using computer aided study.

\section{Methods}

\subsection{Data Sources}

The protein data (SARS-CoV-2 Papain like protease (PLpro) (PDB: 6wx4), Chymotrypsin-like main protease (3CLpro) (PDB:6YB7), SARS-CoV nsp 12 (PDB: 6nus), Host cell protease (TMPRSS1) (PDB:5ce1) were obtained from the Protein Data Bank (PDB), which is a single worldwide depository of information about the 3D structures of large biological molecules, including proteins and nucleic acids (https://www.rcsb.org/structure). The SDS format of each Ligands were obtained from the Pubchem database, the world's largest free chemistry database (https://pubchem.ncbi.nlm.nih.gov) and taken to cactus online smiles translator (https://cactus.nci.nih.gov) for ligand download. Prior to docking analysis, interacting ligands, and water molecules were removed from the protein, thereafter saved in PDB format for docking analysis.

\subsection{Molecular docking and Visualization}

Docking of the proteins with the small molecule inhibitors was performed using Auto Dock Vina 1.5.6. On the Autodock tool, polar-H-atoms were first added to the proteins followed by Gasteiger charges calculation. The protein file was saved as pdbqt file and the grid dimensions were set. Docking calculations (Binding Affinity $(\Delta G)$ were then performed by using Vina folder (Trott and Olson, 2010). Interactions between the ligand and proteins were visualized using discovery studio 2019. 


\subsection{ADMET Profiling}

PubChem database was applied to get the smiles structures of the natural compounds, and was further used for the ADMET prediction. The qualitative assessment of pharmacokinetics viz; absorption, distribution, metabolism, excretion and toxicity (ADMET) profile of selected compounds were predicted computationally by using vNN Web Server for ADMET Predictions (https://vnnadmet.bhsai.org).

\section{Results And Discussion}

\subsection{Preventing viral synthesis and replication}

\subsubsection{Papain like protease (PLpro)}

Cysteine protease papain like protease (PLpro) recognizes a tetra peptide motif found in-between nsp1 and nsp2, nsp 2 and nsp 3, and nsp 3 and nsp 4 proteins that are essential for viral replication (Han et al., 2005; Harcourt et al., 2004; Rut et al., 2020). It has been reported that PLpro exhibits deubiquitination and de-ISGylation; thus blocking the interferon regulatory factor (IRF3) pathway (Lindner et al., 2007; Mielech et al., 2014; Yang et al., 2014). Besides, it inhibits production of cytokines and chemokines responsible for host activation of innate immune response against virus (Clementz et al., 2010; Devaraj et al., 2007; Frieman et al., 2009). Therefore, PLpro is not only essential in viral replication but also significant to antagonize host innate immunity (Chen et al., 2014; Li et al., 2016; Yuan et al., 2016). Consequently, the protein is an important target in antiviral drug design against SARS-COV-2. Forty-seven compounds were docked against SARS-CoV-2 PLpro (PDB: 6wx4). Dexamethasone was also docked as reference drug. The interaction between the natural compounds and the protein is revealed in Table 1 with the binding affinity $(\mathrm{kcal} / \mathrm{mol})$ and calculated inhibition constant $(\mathrm{Ki} \mu \mathrm{m})$.

Twenty-four of the compounds have binding affinity $\left(\Delta \mathrm{G}^{\circ}\right) \leq-6.0 \mathrm{Kcal} / \mathrm{mol}$, out of forty-seven docked compounds whereas Dexamethasone had binding affinity of $-7.1 \mathrm{Kcal} / \mathrm{mol}$.

Quercetin 3,7,4-triglucoside (-8.6 Kcal/mol), Quercetin-7,4-diglucoside (-8.5 Kcal/mol), Quercetin 3,4diglucoside $(-8.2 \mathrm{Kcal} / \mathrm{mol})$, Quercetin-4-glucoside $(-8.2 \mathrm{Kcal} / \mathrm{mol})$, Isohamnetin-4-diglucoside $(8.1$ $\mathrm{Kcal} / \mathrm{mol})$, Quercetin-3-glucoside $(-7.7 \mathrm{Kcal} / \mathrm{mol})$, Rutin $(-7.7 \mathrm{Kcal} / \mathrm{mol})$, Isohamnetin 3,4-diglucoside $(7.5$ $\mathrm{Kcal} / \mathrm{mol})$, Kaempferol-3-0-rutinside $(-7.3 \mathrm{Kcal} / \mathrm{mol})$ and Nimbolide $(-7.3 \mathrm{Kcal} / \mathrm{mol})$ displayed stronger binding affinity than dexamethaxone. Though, the binding affinity of Nimbanal $(-7.1 \mathrm{Kcal} / \mathrm{mol})$, Quercetin $(-7.1 \mathrm{Kcal} / \mathrm{mol})$, Apigenin $(-7.0 \mathrm{Kcal} / \mathrm{mol})$, Luteolin $(-7.0 \mathrm{Kcal} / \mathrm{mol})$ and Regorafenib $(-7.0 \mathrm{Kcal} / \mathrm{mol})$ are comparable to that of dexamethasone (Table 1). All the compounds docked in this study have been identified in either Allium cepa, Azadirachta indica or Xylopia aethiopica. Quercetin 3-glucoside, Quercetin 7,4-diglucoside, Kaempferol-3-0-rutinside, Quercetin 3,7,4-triglucoside, Quercetin, Quercetin 3,4-

diglucoside, Isorhamnetin 4-glucoside, Isohamnetin 3,4-diglucoside, Quercetin-4-glucoside. Rutin, Luteolin, Isorhamnetin, Apigenin are Allium cepa isolates while Azadironic acid, Meliacinin, Nimbionone, Nimbanal, Azadirachtin, Regorafenib are Azadirachta indica isolates. Only Cubebene is an isolate from Xylopia aethiopica. Most of the compounds that have stronger inhibition for PLpro were Allium cepa isolates. 
Interactions of the natural compounds with the amino acids at the catalytic site of CoV-2-PLpro was mediated via hydrophobic and hydrogen bond. Alpha terpineol binds within the pocket of SAR-Cov-2PLpro by forming hydrogen bond with Tyr 268, Alkyl and Pi-Alkyl interaction with Tyr 264, Pro 248 and Pro 247 and van der Waals with Asp 164, Gly 163, Tyr 273, Asn 267, and Thr 301. Azadirachtin also interacted with amino acids within the binding pocket. It formed hydrogen bond with Glu 161, Gly 163, Lys 157, Tyr 268, Asp 164 and hydrophobic interaction (van der Waals) with Glu 167, Gln 269, Leu 162, Tyr 273, Tyr 264, Met 208, Thr 301, Pro 247, Pro 243, and Arg 166. Furthermore, Buoebenone interacted with Pro 248, Pro 247 and Tyr 268 via Alkyl and Pi-Alkyl interaction and via Pi-Sigma interaction with Tyr 264 but made van der Waals contact with Thr 301, Tyr 273, Asp 164 and Met 208 within the binding pocket (Supplementary file).

Similarly, Cycloallin interacted with Thr 301, Tyr 268, Gly 163, and Pro 248 via hydrogen bond but with Pro 247, Asp 164, Tyr 273, and Tyr 264 via van der Waals. Pocket amino acids involved in the binding of Isorhamnetin mostly interacted via van der Waals contact, only Leu 162 formed hydrogen bond interaction while Asp 164 formed Pi-Anion interaction (Supplementary file). Binding of Luteolin with the pocket atoms was via hydrophobic interactions; Pro 247, Tyr 268, and Pro 248 formed Alkyl and Pi-Alkyl interaction, Tyr 264 formed Pi-Sigma interaction, while Met 208, Thr 301, Tyr 273, and Asp 164 formed van der Waal contact. Meliacinin interacted with amino acids close to the pocket atoms; it formed hydrogen interaction with Arg 166 and Met 208, it formed Alkyl interaction with Met 206 and Val 202 while it formed van der Waals contact with GIn 174, Tyr 171, Glu 203, Ser 170, and Tyr 207. Myrtenal interacted via Alkyl and Pi-Alkyl interaction with Tyr 273, Pro 248, Tyr 264, and Pro 247 and via hydrogen interaction with Thr 301. It formed van der Waal contact with Tyr 268, Asp 164, Arg 166, Ala 246, and Met 208. Nimbanal formed both hydrogen and hydrophobic interaction with amino acids within the pocket. Thr 301, Tyr 273, and Arg 166 were involved in hydrogen bond interaction; Leu 162 and Tyr 264 were involved in Pi-Sigma interaction and Pro 247 and Pro 248 in Alkyl interaction. Glu 167, Met 208, Ala 246, Asp 164, Gly 163, Tyr 268, and GIn 269 were involved in van der Waals contact. Nimbionone, Quercetin 3glucoside s-propylcysteine and Thymol all interacted with some catalytic atoms of CoV-2-PLpro.

Dexamethasone did not interact with the pocket atoms but were involved in one or more interactions with atoms close to the catalytic site. It interacted with Asn 146, Ala 145, Ala 144, Glu 143, Trp 93, Gly 142 via van der Waals contact, Alkyl interaction with Lys 94 and hydrogen bond with Tyr 95.

Our result showed the natural compounds with high binding affinity for PLpro and probable drug likeliness as revealed in Table 5. Similar to Dexamethasone ADMET prediction revealed that Azadironic acid, Luteolin and Nimbionone among the other potent compounds showed no-toxicity potential viz; no drug induced liver injury (DILI), mutagenic (AMES) and cytotoxic likeliness. These compounds are as well non-inhibitor to drug metabolizing enzymes. It is important to note that, several of the compounds with anti-viral potentials were predicted to either have DILI, cytotoxicity and mutagenic (AMES) likeliness. For instance, Apigenin was predicted to be non-toxic but could be inhibitor of different isoforms of drug metabolizing enzymes, 3-deacetylsalanin, Cubebene, Kaempferol-3-0-rutinside, Meliacinin and Azadirachtin showed DILI likeliness. Isorhamnetin, Isorhamnetin 4-glucoside, Isohamnetin 3,4diglucoside, Quercetin 3-glucoside, Quercetin 7,4-diglucoside and Quercetin 3,7,4-triglucoside were 
predicted to have mutagenic likeliness. Overall, similar to dexamethasone, our study showed that among the compounds with strong affinity for PLpro, only Azadironic acid, Nimbionone and Luteolin significant pharmacokinetic properties probable for drug development.

\subsubsection{Chymotrypsin-like main protease (3CLpro)}

These compounds from Azadirachta indica, Allium cepa and Xylopia aethiopica were also docked against the SAR-CoV-2 viral replicating protease; Chymotrypsin-like main protease (PDB: 6YB7). Chymotrypsin-like protease together with PLpro is involved in the proteolytic processing of viral polyprotein that are translated from the viral RNA (Hilgenfeld, 2014). It cleaves at not less than 11 conserved sites on the large polyprotein (Hegyi and Ziebuhr, 2002; Zhang et al., 2020). The 3CLpro is an important drug target because of its important role in viral life cycle (Pillaiyar et al., 2016). During its cleavage function on viral polyprotein, it has a unique recognition sequence (Leu-GIn $\downarrow$ (Ser,Ala,Gly $\downarrow$ marks the cleavage site) that is not recognized by any known human protease (Zhang et al., 2020). Therefore, compounds capable of inhibiting the protein are unlikely to be toxic. The binding affinity of the compounds was between $-3.9 \mathrm{Kcal} / \mathrm{mol}$ (for s-propylcysteine) and $-10.8 \mathrm{Kcal} / \mathrm{mol}$ (for Quercetin 3,7,4triglucoside) (Table 2). To classify the compounds as potent, those with binding affinity $\geq-8.0 \mathrm{Kcal} / \mathrm{mol}$ were noted and regarded as having good binding affinity for 3CLpro. In this regard, twenty three of the compounds; Quercetin 3,7,4-triglucoside $>$ Kaempferol-3-0-rutinside $>$ Rutin $>$ Quercetin 3,4-diglucoside $=$ Isohamnetin 3,4-diglucoside > Quercetin-7,4-diglucoside > Quercetin-4-glucoside $=$ Regorafenib $>$ Quercetin-3-glucoside $>$ Isorhamnetin-4-glucoside $>$ Azadironic acid $=$ Nimbolide $>$ Nimbanal $=$ Luteolin $=$ Meliacinin $=$ Nimocinol $>$ Apigenin $>$ Quercetin $>$ Nimbionone $>$ 3-deacetylsalanin $>$ Azadirachtin $=$ Isorhamnetin = Nimbionol could serve as potential drug candidate for the management of SARS-COV-2. In addition, Quercetin and Isorhamnetin with their derivatives showed unique characteristics in their affinity for 3CLpro as revealed by the binding affinity and inhibition constant (Table 2). The affinity of the derivatives for the viral protein increased with the number of glucoside chain.

Visualization of interactions between the ligands and the protein showed that majority of the compounds with good binding affinity for the protein formed either Alkyl or Pi-Alkyl interaction with Ala 285 and/or Leu 286. The two residue are involved in the catalytic activity of the protease (Lim et al., 2014) and interaction between domain II and III of the main protease. 3-deacetylsalanin and Apigenin interacted with Leu 286 via alkyl and Pi-Alkyl interaction respectively. Isohamnetin 3,4-diglucoside and Azadirachtin formed one or more covalent hydrogen bond with Leu 287, Tyr 239, Lys 137, Thr 199 or Tyr 237 but Alkyl interaction with Leu 286, other interacting amino acids were involved in van der Waal interaction.

Dexamethasone, Azadironic acid, Citral, Cubebene, Methychavicol and Nimocinol were involved in one or more Alkyl or Pi-Alkyl interactions with Leu 286, Leu 287, Met 276, Leu 272, Tyr 237 and Tyr 239. Van der Waal interaction existed between Luteolin and Leu 286 but Pi-Alkyl interaction with Leu 287. For Nimbanal, covalent hydrogen bond was formed with residue Asp 197, Lys 137 and Arg 131, Pi-Sulfur interaction with Met 276, Pi-Alkyl interaction with Leu 286 and Pi-Sigma interaction with Leu 287 while Nimbionone interacted with Leu 286 via Pi-Sigma interaction. Quercetin 3,4-diglucoside formed a covalent interaction with Glu 290, Glu 288, Thr 199 and Asn 238 but Pi-Sigma interaction with Leu 286. 
The conventional hydrogen bond interaction with Quercetin 3,7,4-triglucoside existed with residue Asp 197, Lys 137, Asn 238, Thr 199, Tyr 237, Lys 5, Glu 288, Leu 272 and Gly 275, van der Waal interaction was formed with Ala 285 and Pi-Alkyl interaction with Leu 286 and 287. For Quercetin 3-glucoside, hydrogen bond interaction was formed with residue Tyr 237, Asp 197 and Glu 290 but Pi-Alkyl interaction with Leu 286. The covalent hydrogen bond between the protein and Quercetin 7,4-diglucoside existed with residue Arg 4, Lys 5, Ser 284, Leu 282, Leu 287, Arg 131 and Asp 289 while interaction with Leu 286 was via van der waal contact. For Quercetin, the hydrogen bond interaction was with residue Asp 289 and Glu 288, but a Pi-Sigma interaction with Leu 286 and Pi-Alkyl with Leu 287. Covalent hydrogen bond in the binding interaction of Quercetin-4-glucoside with the protein existed in the residue Lys 137, Asp 289, Glu 288, but the Pi-Alkyl interaction was with Leu 286 and 287. Rutin formed covalent hydrogen interaction with residue Asn 238, Lys 236 and Asp 289 but Pi-Alkyl interaction with Leu 286 and 287 (Supplementary file). Salanone acetate formed hydrogen bond with Lys 137 and carbon hydrogen bond with Asp 197, while it interacted with Leu 286 via Alkyl interaction. The ADMET prediction of the compounds showed that all the compounds have probable drug potentials relative to the standards, however, only Azadironic acid, Luteolin, Nimbionone, Nimbolide, Nimbionol, Nimocinol were predicted to have no toxicity likeliness (Table 5).

\subsubsection{RNA-dependent RNA polymerase (RdRp)}

The 932 amino acid (a.a.) SARS-CoV nsp 12 (PDB: 6nus) was docked against the 47 natural compounds and Dexamethasone. The protein is a RNA-dependent RNA polymerase having its polymerase domain between a.a 398-919 (McDonald et al., 2013). It is a vital enzyme for coronavirus replication. In complex with nsp 7 and nsp 8 as co-factors, this protein is greatly stimulated to perform polymerase activity (Ahn et al., 2012; Subissi et al., 2014). Homology studies revealed that the SARS-COV nsp 12 shared several kinase catalytic residues with protein kinases (Kirchdoerfer and Ward, 2019) therefore suggesting potential nucleotide-binding site within the enzyme domain and nucleotidyl transfer (Lehmann et al., 2015) function. The detail understanding of the mechanism of activity of the SAR-COV RdRp is yet a big complex to be unraveled. Both the binding affinity and the inhibition constant were factors in the selection of the most potent of the natural compounds. Twenty-seven (27) of the compounds including Cubebene, Copaene and Buoebenone having binding affinity from $-6.0 \mathrm{Kcal} / \mathrm{mol}$ and above and inhibition constant from $39.3 \times 10^{-6}(\mu \mathrm{M})$ below showed similar anti-viral property with Dexamethasone by inhibiting SARS-CoV RNA-dependent RNA polymerase. The order of drug's affinity for RdRp includes; Nimocinol> Quercetin 3,4-diglucoside> Azadironic acid> Quercetin 7,4-diglucoside> Quercetin 3,7,4triglucoside>Quercetin 3-glucoside>Isohamnetin 3,4-diglucoside>Rutin > Quercetin-4glucoside>Isorhamnetin 4-glucoside=Kaempferol-3-0-rutinside $>$ Regorafenib $>$ Luteolin= Nimbolide $>$ Apigenin $>$ Nimbionone $=$ Quercetin $=$ Salannol acetate $>$ Nimbanal $>$ Azadirachtin $=$ Isohamnetin3,4diglucoside $>$ Nimbionol $>$ Copaene $=$ Isorhamnetin $>3$ deacetylsalanin>Buoebenone>Cubebene (Table 3). Isorhamnetin and it glucoside derivative showed higher affinity and lower inhibition constant when the number of glucose moiety increased in the side chain. All the compounds interacted with the protein within the polymerase domain. The polymerase 
domain is comprised of a fingers domain a.a. 398-581, 628-687), a palm domain (a.a. 582-627, 688815), and a thumb domain (a.a. 816-919). (Kirchdoerfer and Ward). Bornyl acetate was well fitted within the acceptor template nucleotide position of RdRp where it formed hydrogen bond with Asn 496, Asn 497 and Arg 569. The compound formed Alkyl and Pi-Alkyl interaction with Tyr 689, Leu 576 and Ala 685, but van der Waal interaction with Lys 577 (Supplementary file). This position was reported to be the binding pocket of remdesivir (Wu et al., 2020). Nimbanal formed hydrogen bond interaction with Tyr 689, Asn 497, Arg 569 ans Lys 500. Carbon hydrogen bond was also formed with Ser 682, Alkyl, and Pi-Alkyl interaction with Ala 688 (Supplementary file). Quercetin 3-glucoside binds with amino acids within the palm domain. It interacted via hydrogen bond with Ser 759, Ala 762, and Trp 617, Pi-Alkyl interaction with Leu 758 and Carbon hydrogen bond with Cys 813. Ala 688, Gly 616, Trp 800, Lys 798, Asp 760 all interacted via van der Waals. Gamma-s-propyl-cysteine interacted with amino acid residue close to the acceptor template nucleotide position; it formed hydrogen bond with Gln 541, Val 560, Gly 683, Thr 540 and Glu 665. It formed van der Waal interaction with Gly 503, Met 542, Tyr 456, Val 667, Ser 682 anD Ser 681. Isohamnetin 3,4-diglucoside formed hydrogen bond interaction with Thr 710, Gly 712, Arg 721 and Tyr 732, Pi-Pi Stacked interaction with Tyr 728, Pi-Alkyl interaction with Leu 708 and carbon hydrogen bond with Asp 711 and Leu 708. The interactions with Ser 709, Tyr 129, His 133, Asn 705, Leu 731, Leu 240 existed via van der waals. Isorhamnetin 4-glucoside formed Hydrogen bod interaction with His 133, Asp 711, Gly 712 and Gln 724. Pi-Alkyl interaction was formed with Val 128 and Leu 708, Pi-Sigma with Leu 240 and van der Waal with Thr 710. Dexamethasone interacted with Thr 710 and Ser 709 via hydrogen bond interaction, and with Tyr 129, Leu 240 and ALA 125 via Alkyl and Pi-Alkyl interaction.

Similar to observation in the docking of the other viral proteins reported in this study, the 27 most potent of the natural compounds docked with RdRp have probable drug potentials as indicated in the ADMET prediction (Table 5). Azadironic acid, Buoebenone, Luteolin, Nimbionone, Nimbolide, Nimbionol and Nimocinol showed unique potentials as anti-viral drug with no toxicity likeliness.

\subsection{Targets blocking host specific enzymes}

\subsubsection{Host cell protease}

The ligands were docked against the Hepsin serine protease (PDB: 5ce1), which is a host type II transmembrane serine protease (TTSP), responsible for the activation cleavage for SARS-CoV spike (Lennart et al., 2017). The catalytic sites of diverse serine proteases linked to parainfluenza, influenza and coronavirus activation are structurally similar; therefore, active site inhibitors of this protein could have broad applicability against multiple respiratory viruses (Laporte and Naesens, 2017). Twenty-three of the docked compounds showed good binding affinity with the protein similar to dexamethaxone. Compounds with binding affinity $\leq-6.5 \mathrm{Kcal} / \mathrm{mol}$ were included among the compounds (Table 4). Dexamethasone binds to the pocket of the protein where they exhibited different forms of interactions within the catalytic traid (His 203, ASP 257, and Ser 353). Quercetin 7,4-diglucoside, Isorhamnetin 4-glucoside, Isorhamnetin 3,4-diglucoside, Quercetin 3-glucoside, Quercetin 4-glucoside Quercetin, Nimocinol and Kaempferol-3-Orutinside formed Pi-Cationionic interaction with His 203. Other interactions such as Pi-Sigma, Pi-Pi T- 
shaped and Pi-Alkyl were formed with His 203 by Nimocinol, Pi-Alkyl interactions were also formed with Trp 377 and Tyr 301, Ser 353 and other residues formed van der Waal interactions with Nimocinol. Quercetin 7,4-diglucoside formed van der Waal interaction with Ser 353 and conventional hydrogen bond interaction with Gly 380, Cys 381, Ala 348, Trp 377, Tyr 187 and Asn 209. Kaempferol-3-0-rutinside on the other hand formed conventional hydrogen bond interaction with Ser 353, Gly 378, Asn 250, Tyr 243 and Leu 187 (Supplementary file), while Isorhamnetin 4-glucoside formed Pi-Alkyl interaction with Pro 206, but conventional hydrogen bond interaction with Asn 250, Gly 380 and Gly 378. Conventional hydrogen bond formed by Isorhamnetin 3,4-diglucoside were with residue Cys 381, Ala 348, Gly 378, and Gly 380, van der waal interactions were formed with residue Ser 353, Pro 206 and the other amino acids (Table 4). Two benzene rings in Rutin formed Pi-Cationionic and Pi-Pi T-shaped interactions with His 203, and Hydrogen, and Pi-Cationic interaction with Arg 208. Conventional hydrogen bond interaction was only formed with Ser 353 by Quercetin 3-glucoside, other interactions included Amide-Pi Stacked with Cys 349, Pi-Alkyl with Cys 381, while other residues interacted via van der Waals. Quercetin formed hydrogen bond with Asp 347 and Ala 348, Pi-Pi Stacked and Amide Pi-Stacked with Cys 349 and Trp 377, Pi-Alkyl with Cys 381 and Carbon hydrogen bond with Trp 377. Quercetin 3, 4-diglucoside, and Regorafenib formed conventional hydrogen bond interaction with His 203, Ser 353, Asn 209, Asn 298, Gln 350 and Gly 378. Salannol acetate formed it hydrogen bond interaction with His 203 and Ser 353, Amide-Pi-Stacked interaction with Ser 376, Alkyl and Pi-Alkyl interactions with Ala 348, Tyr 301, Cys 381, Trp 377 and His 203 , other residues interacted via van der Waals interaction. Conventional hydrogen bond interaction existed for Apigenin on residues His 203, Ala 348, Asp 347 and Gln 350, Carbon hydrogen bond interaction with Gly 388, Amide Pi-Stacked interaction was formed with Trp 377 and Cys 349, Pi-Alkyl interaction was formed with Cys 381, Ser 353 while other residues exhibited van der Waal interactions with the ligand. Nimbanal formed hydrogen bond interaction with Ser 353, His 203, Gly 351, Tyr 243, Asn 250. Alkyl and Pi-Alkyl interactions were formed with His 203 and Pro 206 on different rings of Nimbanal. Conventional hydrogen bond interactions formed with Nimbolide were with Gly 351, Gln 350 and Ser 353, Alkyl and Pi-Alkyl interactions existed with His 203, Leu 187 and Ala 185. Only His 203 formed hydrogen bond interaction with Luteolin, Pi-Alkyl interaction was formed with Cys 381, Ala 348 and Amide-Pi Stacked interaction with Trp 377 and Cys 349, Ser 353 and the other residue interacted via van der Waals. Conventional hydrogen bond interaction was formed by Isorhmnetin with His 203, Gly 380 and Ser 353, carbon hydrogen bond also existed with Cys 349, Ser 347 and Ser 376. Nimbionone and Meliacinin bind to a different site other than the catalytic site of Hepsin Serine protease. Nimbionone formed Pi-Sigma interaction with Phe 66, Pi-Alkyl interaction with Leu 51 and carbon hydrogen bond with Pro 53. Meliacinin formed hydrogen bond interaction only with GIn 283, Alkyl and Pi-Alkyl interactions were formed with Pro 53, Leu 51, Trp 73, Phe 66 and Ala 284.

The ADMET prediction showed six of the compounds viz; Azadironic acid, Luteolin, Nimbionone, Nimbolide, Nimbionol and Nimocinol together with Dexamethasone succeeded in all the ADMET prediction parameters (Table 5). Overall, the results of the study indicated that some of the compounds have potentials for multiple target. For instance, Apigenin, Azadirachtin, Azadironic acid, 3deacetylsalanin, Kaempferol-3-0-rutinside, Isorhamnetin, Isohamnetin 3,4-diglucoside, Luteolin, 
Nimbionone, Nimocinol, Nimbolide, Nimbanal, Meliacinin, Quercetin 3,4-diglucoside, Quercetin 3diglucoside, Quercetin 4-diglucoside, Quercetin, Regorafenib, Rutin had good binding affinity for all the docked proteins. It is important to note that majority of this compounds are compounds already identified in either $A$ indica (8) or A. cepa (11).

\subsection{ADMET Profile of Docked Compounds}

Early assessments of pharmacokinetic and toxic properties are important step in drug discovery. Drug discovery is a risky, lengthy, and resource-intensive process, however, toxicity remains a hurdle, that has resulted in attrition of several drugs at the clinical trial stage and even already approved drugs (Waring et al., 2015). Aside efficacy and toxicity, many drug development failures are imputable to poor pharmacokinetics and bioavailability (Darvas et al. 2002; Daina and Zoete, 2016). It is therefore imperative nowadays to design lead compounds with good pharmacokinetics and bioavailability and carefully select compounds during drug development to avoid late-stage attrition. In this study, fortyseven natural compounds and dexamethaxone were assessed for their pharmacokinetics and toxicity properties using in silico approach. According to the pharmacokinetic prediction in Table 5, only Bornyl acetate has ability for blood brain barrier (BBB) penetration. All the compounds were predicted to be stable to the human liver microsomal (HLM). The human liver is the most important organ for drug metabolism. For a drug to achieve effective therapeutic concentrations in the body, the liver cannot metabolize it too rapidly. Otherwise, it would need to be administered at high doses, which are associated with high toxicity (Schyman et al., 2017). In metabolism, most of the compounds appeared to be none inhibitors to P450. However, Salannol acetate and 3-deacetylsalanin could be inhibitor to Cyp3A4, Apigenin could be inhibitor to Cyp1A2 and Cyp2C19, Quercetin and Isorhamnetin could be inhibitor to Cyp1A2 and Cyp2C9, Luteolin could be inhibitor to Cyp1A2, while Nimbanal and Nimbolide could be inhibitor to Cyp3A4. As earlier reported, inhibition of this protein could increase a compound in the plasma and decrease the clearance of its substrate (Flockhart, 2007).

P-glycoprotein (Pgp) is a critical determinant of the pharmacokinetic properties of drugs as it function to extracts foreign substances from the cell (Ambudkar et al., 2003). Cancer cells often overexpress Pgp, consequently increasing the efflux of chemotherapeutic agents from the cell and reducing the effective intracellular concentrations of such agents; multidrug resistance (Borst and Elferink, 2002). It is of great interest to identify if a compound is a substrate to Pgp (can be transported out of the cell) or inhibitor to Pgp (impair function). Majority of the compounds are Pgp substrate while some were predicted as both substrate and inhibitor. The toxicity prediction showed that of all the compounds, only Regorafenib and Verbenone were predicted to have cytotoxicity potential. Isohamnetin 3,4-diglucoside, Isorhamnetin 4glucoside, Isorhamnetin, Quercetin 3,4-diglucoside, Quercetin 3,7,4-triglucoside, Quercetin 3-glucoside, Quercetin, Quercetin 7,4-diglucoside and Rutin could induce bacteria mutation (Ames) and therefore cause drug resistance (Aristyani et al., 2018). The human ether-à-go-go-related gene (hERG) codes for a potassium ion channel involved in the normal cardiac repolarization activity of the heart (Sanguinetti and TristaniFirouzi, 2006). Blockade of hERG function can cause long QT syndrome, which may result in arrhythmia and death (De Ponti et al., 2001); based on the prediction, all the compounds except 
Regorafenib have no cardio toxic potential. Some of the compounds could also induce liver injury (DILI) (Table 5). Ten of the compounds predicted to potentiate mitochondria dysfunction (decrease MMP) was recorded as yes (Table 5). Finally, in Table 5, the maximum recommended therapeutic dose (MRTD) for each compound was predicted and recorded as $\mathrm{mg} /$ day for an average adult with a body weight of $60 \mathrm{~kg}$ (Schyman et al., 2017).

\section{Conclusion}

In this study, the forty-seven isolates from Allium cepa, Azadirachta indica and Xylopia aethiopica that were docked against the viral and host proteins demonstrated negative binding affinity. Among the compounds, it appears Quercetin 3,7,4-triglucoside, Quercetin-7,4-diglucoside, Quercetin 3,4-diglucoside, Quercetin-4-glucoside, Isohamnetin-4-diglucoside, Quercetin-3-glucoside, Rutin, Isohamnetin 3,4diglucoside, Kaempferol-3-O-rutinside, Nimbolide, Nimbanal, Quercetin, Apigenin, Luteolin and Regorafenib could target all the viral proteins studied by exhibiting good binding affinity and thus could serve as ideal inhibitors for SARS-CoV-2. Overall, although many of the compounds have good binding affinity for the viral and host proteins, our study indicated that only Azadironic acid, Nimbionone, Nimbionol and Nimocinol all from A. indica could serve as potential drug candidate with good pharmacokinetics and toxicity profile comparable with dexamethasone. Therefore, our study provides an insight into potential inhibitors and novel drug candidates for SARS-CoV-2. Further studies will determine the dynamics of the interactions of the potent compounds with the proteins.

\section{Declarations}

\section{Conflict of Interest}

The authors declare no existing conflict of interest

\section{References}

Ahmadi, S., Rajabi, Z., Marandi, M.V. (2018). Evaluation of the antiviral effects of aqueous extracts of red and yellow onions (Allium Cepa) against avian influenza virus subtype H9N2. Iranian Journal of Veterinary Science and Technology. 2018-2 (19)

Ahn, D. G., Choi, J. K., Taylor, D. R. \& Oh, J. W. Biochemical characterization of a recombinant SARS coronavirus nsp12 RNA-dependent RNA polymerase capable of copying viral RNA templates.Arch. Virol.157, 2095-2104 (2012).

Ambudkar, S. V., Kimchi-Sarfaty, C., Sauna, Z. E., and Gottesman, M. M. (2003). P-glycoprotein: from genomics to mechanism. Oncogene 22, 7468-7485.doi: 10.1038/sj.onc.1206948

Aristyani S., Nur M.I., Wldyarti S., Sumitro S.B. (2018). In silico study of active compounds ADMET profiling in Curuma xanthorrhiza Roxb and Tamarindus indica as Tuberculosis Treatment. Journal Jsmu 
Borst, P., and Elferink, R. O. (2002). Mammalian ABC transporters in health and disease. Annu. Rev. Biochem. 71, 537-592. doi: 10.1146/annurev.biochem.71.102301.093055

Chen $X$, Yang X, Zheng Y, Yang Y, Xing Y, Chen Z. SARS coronavirus papain-like protease inhibits the type I interferon signaling pathway through interaction with the STING-TRAF3-TBK1 complex. Protein Cell 2014; 5: 369-81.

Chen, C. H., Chou, T. W., Cheng, L. H., Ho, C. W. (2011). In -vitro anti-adenoviral activity of five Allium plants. Journal of the Taiwan Institute of Chemical Engineers, 42(2):228-232.

Clementz, M.A., Chen, Z., Banach, B.S., Wang, Y., Sun, L., Ratia, K., Baez-Santos, Y.M., Wang, J., Takayama, J., Ghosh, A.K., Li, K., Mesecar, A.D., Baker, S.C., 2010. Deubiquitinating and interferon antagonism activities of coronavirus papainlike proteases. J. Virol. 84, 4619-4629

Daina A. and Zoete, V. (2016). ABOILED-Egg To Predict Gastrointestinal Absorption and Brain Penetration of Small Molecules. ChemMedChem 2016, 11,1117- 1121

Darvas F, Keseru G, Papp A, Dormán G, Urge L, Krajcsi P (2002)In Silico and Exsilico ADME approaches for drug discovery. Top Med Chem 2:1287-1304

De Ponti, F., Poluzzi, E., and Montanaro, N. (2001). Organising evidence on QT prolongation and occurrence of Torsades de Pointes with nonantiarrhythmic drugs: a call for consensus. Eur. J. Clin. Pharmacol. 57, 185-209. doi: 10.1007/s002280100290

Devaraj, S. G., Wang, N., Chen, Z., Chen, Z., Tseng, M., Barretto, N., Lin, R., Peters. C.J.Tseng, C.K., Baker, S.C., Li, K. Regulation of IRF-3-dependent innate immunity by the papain-like protease domain of the severe acute respiratory syndrome coronavirus. J Biol Chem 282, 32208-32221 (2007).

Efferth T and Koch E. Complex interactions between phytochemicals. The multi-target therapeutic concept of phytotherapy. Current Drug Targets. 2011; 12(1):122-132.

Faccin-Galhardi, L.C., Yamamoto, K.A., Ray, S., Ray, B., Linhares, R.E.C., Nozawa, C. (2012). The in vitro antiviral property of Azadirachta indica polysaccharides for poliovirus. Journal of Ethnopharmacology 142 (2012) 86-90

Flockhart DA. 2007. Drug Interactions: cytochrome P450 Drug Interaction Table. Indiana University School of Medicine'/clinpharm/ddis/clinical-table/"Accessed August $12^{\text {th }} 2018$

Frieman, M., Ratia, K., Johnston, R.E., Mesecar, A.D., Baric, R.S. Severe Acute Respiratory Syndrome Coronavirus Papain-Like Protease Ubiquitin-Like Domain and Catalytic Domain Regulate Antagonism of IRF3 and NF-kappa B Signaling. J Virol 83, 6689-6705 (2009). 
Han, Y. S. Chang, G.G., Juo, C.G., Lee, H.J., Yeh, S.H., Hsu, J.T., Chen, X. Papain-like protease 2 (PLP2) from severe acute respiratory syndrome coronavirus (SARS-CoV): Expression, purification, characterization, and inhibition. Biochemistry-Us 44, 10349-10359 (2005).

Harazem, R., Rahman, S., Kenawy, A. 2019. Evaluation of Antiviral Activity of Allium Cepa and Allium Sativum Extracts Against Newcastle Disease Virus. Alexandria Journal of Veterinary Sciences, 61(1).

Harcourt, B. H., Jukneliene, D., Kanjanahaluethai, A., Bechil, J., Severson, K.M., Smith, C.M., Rota, P.A., Baker, S.C. Identification of severe acute respiratory syndrome coronavirus replicase products and characterization of papain-like protease activity. J Virol 78, 13600-13612 (2004

Hegyi, A. and Ziebuhr, J. Conservation of substrate specificities among coronavirus main proteases. J. Gen. Virol. 83, 595-599 (2002).

Hilgenfeld, R. From SARS to MERS: Crystallographic studies on coronaviral proteases enable antiviral drug design. FEBS J. 281, 4085-4096 (2014). doi:10.1111/febs.12936

Kirchdoerfer, R.N. and Ward, A.B. (2019). Structure of the SARS-CoV nsp12 polymerase bound to nsp7 and nsp8 co-factors. NATURE COMMUNICATIONS. 10:2342 1-9. https://doi.org/10.1038/s41467-01910280-3

Kumar, S., Pandey, A. K. 2013. Chemistry and Biological Activities of Flavonoids: An Overview. Pages 116

Laporte M. and Naesens, L. (2017). Airway proteases: an emerging drug target for influenza and other respiratory virus infections. Current Opinion in Virology. 24:16-24

Lehmann, K. C., Gulyaeva, A., Zevenhoven-Dobbe, J.C., Janssen, G.M.C., Ruben, M., Overkleeft, H.S., Veelen, P.A., Samborskiy, D.V., Kravchenko, A.A., Leontovich, A.M., Sidorov, I.A., Snijder, E.J., Posthuma, C.C., Gorbalenya, A.E. Discovery of an essential nucleotidylating activity associated with a newly delineated conserved domain in the RNA polymerasecontaining protein of all nidoviruses. Nucleic Acids Res.43, 8416-8434 (2015)

Lennart, M.R., Reinke, M., Spiegel, M., Plegge, T., Hartleib, A., Nehlmeier, I., Gierer, S., Hoffmann, M., Hofmann-Winkle, H., Winkler, M., P€ohlmann, S. Different residues in the SARS-CoV spike protein determine cleavage and activation by the host cell protease TMPRSS2, PloS One 12 (6) (2017), e0179177

Li S.W, Wang C.Y, Jou Y.J, Huang S.H, Hsiao L.H, Wan L, Lin, Y.J., Kung, S.H., Lin, C.W. SARS coronavirus papain-like protease inhibits the TLR7 signaling pathway through removing Lys63-linked polyubiquitination of TRAF3 and TRAF6.Int J Mol Sci 2016; 17: 678.

Lim, L., Shi, J., Mu, Y., Song, J. Dynamically-driven enhancement of the catalytic machinery of the SARS 3C-like protease by the S284-T285-I286/A mutations on the extra domain. PLOS ONE 9, e101941 (2014) 
Lindner, H.A., Lytvyn, V., Qi, H., Lachance, P., Ziomek, E., Menard, R. Selectivity in ISG15 and ubiquitin recognition by the SARS coronavirus papain-like protease. Arch Biochem Biophys 466, 8-14 (2007).

McDonald, S. M. RNA synthetic mechanisms employed by diverse families of RNA viruses. Wiley Interdiscip. Rev. RNA4, 351-367 (2013).

Mielech, A. M., Kilianski, A., Baez-Santos, Y. M., Mesecar, A. D., and Baker, S. C. (2014) MERS-CoV papainlike protease has delSGylating and deubiquitinating activities.Virology 450-451,64-70.

Parida, M.M., Upadhyay, C., Pandya, G., Jana, A.M. Inhibitory potential of neem (Azadirachta indica Juss) leaves on dengue virus type-2 replication, J. Ethnopharmacol. 79 (2002) 273-278

Pillaiyar, T., Manickam, M., Namasivayam, V., Hayashi, Y. \& Jung, S. H. An overview of severe acute respiratory syndrome-coronavirus (SARS-CoV) $3 \mathrm{CL}$ protease inhibitors: peptidomimetics and small molecule chemotherapy. J. Med. Chem. 59, 6595-6628 (2016).

Rut, W., Lv, Z., Zmudzinski, M., Patchett, S., Nayak, D., Snipas, S.J., Oualid, F.E., Huang, T.T., Bekes, M., Drag, M., Olsen, S.K. (2020). Activity profiling and structures of inhibitor-bound SARS-CoV-2-PLpro protease provides a framework for anti-COVID-19 drug design. BioRxIV preprint.

https://doi.org/10.1101/2020.04.29.068890

Sanguinetti, M. C., and Tristani-Firouzi, M. (2006). hERG potassium channels and cardiac arrhythmia. Nature 440, 463-469. doi: 10.1038/nature04710

Schyman P, Liu R, Desai V and Wallqvist A (2017) vNN Web Server for ADMET Predictions. Front. Pharmacol. 8:889. doi: 10.3389/fphar.2017.00889

Subissi, L., Posthumja, C.C., Collet, A., Zevenhoven-Dobbe, J.C., Gorbalenya A.E., Decroly E., Snijder, E.J., Canard, B., Imbert, I. One severe acute respiratory syndrome coronavirus protein complex integrates processive RNA polymerase and exonuclease activities. Proc. Natl. Acad. Sci. USA111, E3900-E3909 (2014).

Trott, O., Olson, A. J. (2010). AutoDock Vina: improving the speed and accuracy of docking with a new scoring function, efficient optimization and multithreading, Journal of Computational Chemistry 31: 455461

Waring, M. J., Arrowsmith, J., Leach, A. R., Leeson, P. D., Mandrell, S., Owen,R. M., et al. (2015). An analysis of the attrition of drug candidates from four major pharmaceutical companies. Nat. Rev. Drug Discov. 14, 475-486. doi: 10.1038/nrd4609

WHO (2020). WHO Coronavirus Disease (COVID-19) Dashboard. Accessed 27/07/2019, 7: 24 pm CEST. Available online, https://covid19. who.int/?gclid=EAlalQobChMlp-

a2oLLu6gIVB7LtCh00eQGkEAAYASAAEgJJcfD_BwE 
Wu, C., Liu, Y., Yang, Y., Zhang, P., Zhong, W., Wang, Y., Wang, Q., Xu, Y., Li, M., Li, X., Zheng, M., Chen, L., Li, H. (2020). Analysis of therapeutic targets for SARS-CoV-2 and discovery of potential drugs by computational methods. Acta Pharmaceutica Sinica B. https://doi.org/10.1016/j.apsb.2020.02.008

Xu, J., Song, X., Yin, Z.Q., Cheng, A.C., Jia, R.Y., Deng, Y.X., Ye, K.C., Shi, C.F., Lv, C., Zhang, W. (2012). Antiviral activity and mode of action of extracts from neem seed kernel against duck plague virus in vitro. Poultry Science. 91: 2802-2807. http://dx.doi.org/ 10.3382/ps.2012-02468

Yang, X., Chen, X., Bian, G., Tu, J., Xing, Y., Wang, Y., and Chen, Z. (2014) Proteolytic processing, deubiquitinase and interferon antagonist activities of Middle East respiratory syndrome coronavirus papain-like protease.J. Gen. Virol. 95, 614-626.

Yuan L, Chen Z, Song S, Wang S, Tian C, Xing G., Chen, X., Xiao, Z.X., He, F., Zhang, L. p53 degradation by a coronavirus papain-like protease suppresses type I interferon signaling. J Biol Chem 2015; 290: 317282.

Zhang, J., Lin, D., Sun, X., Curth, U., Drosten, C., Sauerhering, L., Becker, S., Rox, K., Hilgenfeld, R. (2020). Crystal structure of SARS-CoV-2 main protease provides a basis for design of improved a-ketoamide inhibitors. Sscience. 1.8

Zong A, Cao, $\mathrm{H}$ and Wang $\mathrm{F}$. Anticancer polysaccharides from natural resources: a review of recent research. Carbohydrate Polymers. 2012; 90(4):1395-1410.

\section{Tables}

Due to technical limitations, tables 1-6 are only available as a download in the supplemental files section.

\section{Supplementary Files}

This is a list of supplementary files associated with this preprint. Click to download.

- Manuscriptcovid2Tables.docx

- GraphicalAbstract..png 